\title{
LAPLACE-TYPE INTEGRAL REPRESENTATIONS OF THE GENERALIZED BESSEL FUNCTION AND OF THE DUNKL KERNEL OF TYPE $B_{2}$
}

\author{
BÉCHIR AMRI AND NIZAR DEMNI
}

\begin{abstract}
In this paper, we derive a Laplace-type integral representations for both the generalized Bessel function and the Dunkl kernel associated with the rank-two root system of type $B_{2}$. The derivation of the first one elaborates on the integral representation of the generalized Bessel function proved in 11 through the modified Bessel function of the first kind. In particular, we recover an expression of the density of the Duistermaat-Heckman measure for the dihedral group of order eight. As to the integral representation of the corresponding Dunkl kernel, it follows from an application of the shift principle to the generalized Bessel function.
\end{abstract}

\section{INTRODUCTION}

Dunkl operators were introduced by C. F. Dunkl in the late eightees and form a commutative algebra of differential-difference operators ([13]). They give rise to the so-called Dunkl kernel which extends the exponential kernel to finite reflection groups and multiplicity functions, and served together with their trigonometric analogues to prove the integrability of Calogero-Moser-Sutherland systems (see e.g. [5, Ch.I). They also allow for a rich harmonic analysis which reduces for special multiplicity values to the one on Cartan motion groups associated with semi-simple Lie groups ([5], Ch.I). The key tool of this analysis is the so-called generalized Bessel function (hereafter GBF) which generalizes the spherical functions on Euclidean-type symmetric spaces. At the probabilistic side, the Dunkl-Laplace operator is the infinitesimal generator of a Feller process referred to as the Dunkl process ([5, CH. II and III). The latter as well as its projection on the positive Weyl chamber were extensively studied and exhibits very interesting properties extending those satisfied by the Brownian motion on Euclidean spaces or reflected in Weyl chambers, and by Bessel processes (see also [9, [12). In particular, the definition of the Duistermaat-Heckman measure associated with a co-adjoint orbit of a compact semi simple Lie group makes now sense for any finite Coxeter group ([3]). Moreover, this measure encodes the Laplace-type integral representation of the GBF when all the multiplicity values equal one. For arbitrary multiplicity values with nonnegative real part, a Laplacetype integral representation still holds and the representative measure is supported in a convex polytope ([6], Corollary 3.3). For $A$-type root systems, its explicit description is obtained in [1] using a contraction principle and induction. There, the polytope supporting the representative measure is the image of the top-row of the $A$-type Gelfand-Tsetlin graph by an affine transformation. Note that this graph as well as its analogues of types $B, C, D$ arise in the study of radial parts of minors of Hermitian random matrices over division algebras (see 7]). However, beware that only the eigenvalues of complex Hermitian ones considered in [7] fit into the theory of Dunkl operators. On the other hand, the Dunkl kernel is also positive-definite (21]) and few attempts to derive its Laplace-type integral representation have been done in [14, 15, 2. Both papers [14] and 2] deal with the $A_{2}$-type Dunkl kernel where its integral representation is deduced from that of the corresponding GBF using the shift principle. Nonetheless, the latter improves the former since it is valid for a larger set of multiplicity values and has less integration variables. As to [15, it deals with the $B_{2}$-type root system and equal multiplicity values. There, the strategy of derivation of the integral representation of the Dunkl kernel is very similar to the one followed in [14] and the obtained formula involves five integration variables.

In this paper, we derive a Laplace-type integral representation of the GBF of type $B_{2}$ over $\mathbb{R}^{2}$ valid for arbitrary multiplicity values whose sum is greater than $1 / 2$. Our derivation starts from the integral representation of the GBF obtained in 11] and is far from being a direct consequence of it. Actually,

Key words and phrases. Dunkl kernel; Generalized Bessel function; Laplace-type integral representation; DuistermaatHeckman measure. 
it relies on a tricky way of writing a six-variables polynomial as the squared Euclidean norm of a planar vector together with an integral representation of the modified Bessel function we prove below. Moreover, the representative measure is shown to be supported in the convex hull of either of both variables as it is supposed to be by the virtue of Corollary 3.3 in [6. When both multiplicity values are equal to one, we get an explicit expression of the Duistermaat-Heckman measure associated with the dihedral group of order eight. Afterwards, we use the shift principle to derive a Laplace-type integral representation for the Dunkl kernel of type $B_{2}$, which is technical and a bit lengthy. Nonetheless, we believe that our resulting formula is optimal in the sense that it as most compact as possible.

The paper is organized as follows. In the next section, we recall some facts on Dunkl operators and the integral representation of the GBF of type $B_{2}$ proved in [11. In the third section, we derive its Laplace-type integral representation and deduce the Duistermaat-Heckman measure associated with the dihedral group of order eight. The fourth section is devoted to the application of the shift principle and to the technical derivation of the Laplace-type integral representation of the Dunkl kernel of type $B_{2}$.

\section{Root systems, the Dunkl kernel and the generalized Bessel function}

For facts on root systems and on Dunkl operators, we refer the reader to the monograph [17]. Let $(V,\langle\cdot, \cdot\rangle)$ be a Euclidean space of dimension $N$ and denote by $\|\cdot\|:=\langle\cdot, \cdot\rangle^{1 / 2}$ the corresponding Euclidean norm. A root system $R$ in $V$ is a finite set of vectors (roots) in $V \backslash\{0\}$ such that

$$
\forall \alpha \in R, \quad \sigma_{\alpha}(R)=R,
$$

where $\sigma_{\alpha}$ is the reflection with respect to $\alpha^{\perp}$ :

$$
\forall x \in V, \quad \sigma_{\alpha}(x)=x-2 \frac{\langle\alpha, x\rangle}{\langle\alpha, \alpha\rangle} \alpha .
$$

It is reduced if

$$
\forall \alpha \in R, \quad R \cap \mathbb{R} \alpha=\{ \pm \alpha\} .
$$

The set $\left\{\sigma_{\alpha}, \alpha \in R\right\}$ generates a finite group $W$, called the reflection group associated with $R$, which acts on $R$ in a natural way. With this action in hands, a $W$-invariant function $k: R \rightarrow \mathbb{C}$ is called a multiplicity function. Therefore, $k$ takes as many values as the number of orbits of $R$ under the action of $W$. Now, we can split $R$ into a positive and a negative parts $R_{+}$and $R_{-}$by choosing a non zero vector $v \in V \backslash R$ and to every choice of positive system $R_{+}$, there exists a unique set $S$ of indecomposable roots referred to as the simple system. The elements of $S$ are the so-called simple roots and characterize $R_{+}$as the set of roots that are linear combinations of simple roots with non negative coefficients.

Now, given a data $\left(R, R_{+}, k\right)$, the Dunkl operator or derivative $T_{\xi}$ in the direction $\xi \in V \backslash\left\{0_{V}\right\}$ is defined by:

$$
T_{\xi}(f)(x)=\partial_{\xi}(f)(x)+\sum_{\alpha \in R_{+}} k(\alpha)\langle\alpha, \xi\rangle \frac{f(x)-f\left(\sigma_{\alpha} x\right)}{\langle\alpha, x\rangle} .
$$

The Dunkl operators form a commutative algebra and if $\left(e_{1}, \ldots, e_{N}\right)$ is the canonical basis of $V$, then we shall simply write $T_{i}$ for $T_{e_{i}}, 1 \leq i \leq N$. Moreover, for generic values of $k$ (for instance those with non negative real parts), there exists a kernel

$$
(x, y) \in V \times V \mapsto D_{k}(x, y)
$$

named the Dunkl kernel, which is the unique analytic solution of the spectral problem:

$$
\begin{aligned}
T_{\xi} D_{k}(\cdot, y)(x) & =\langle\xi, y\rangle D_{k}(x, y), \quad \text { for all } \xi \in V \in \backslash\left\{0_{V}\right\}, \\
D_{k}\left(0_{V}, y\right) & =1, \quad \text { for all } y \in V .
\end{aligned}
$$

This kernel is even positive definite:

$$
D_{k}(x, y)=\int e^{\langle x, z\rangle} \mu_{y}^{(k)}(d z)
$$

for some probability measure $\mu_{y}^{(k)}$ supported in the convex hull $\operatorname{co}(y)$ of the orbit $W y$. More generally, this measure defines the so-called Dunkl intertwining operator which intertwines the algebras of Dunkl and of 
partial derivatives. Now, the generalized Bessel function is defined as the projection of $D_{k}$ onto the space of $W$-invariant functions:

$$
D_{k}^{W}(x, y):=\frac{1}{|W|} \sum_{w \in W} D_{k}(x, w y)=\frac{1}{|W|} \sum_{w \in W} D_{k}(w x, y)
$$

The root system we shall be dealing with in this paper is of type $B_{2}$ and is defined by:

$$
R=\left\{ \pm e_{1}, \pm e_{2}, \pm e_{1} \pm e_{2}\right\} \subset V=\mathbb{R}^{2},
$$

The corresponding reflection group is isomorphic to the dihedral group of order eight:

$$
W=\left(\mathbb{Z}_{2}\right)^{2} \rtimes S_{2}
$$

and the action of $W$ on $R$ splits the latter into two orbits

$$
\left\{ \pm\left(e_{1} \pm e_{2}\right)\right\} \quad\left\{ \pm e_{1}, \pm e_{2}\right\}
$$

to which we attach two multiplicity values $k_{1}, k_{2}, \in \mathbb{R}_{+}^{2}$ respectively. In this case, it was proved in [11] that:

$$
D_{k}^{W}(x, y)=c_{k} \int_{-1}^{1} \int_{-1}^{1} \mathcal{I}_{\gamma-1 / 2}\left(\sqrt{\frac{Z_{x, y}(u, v)}{2}}\right)\left(1-u^{2}\right)^{k_{1}-1}\left(1-v^{2}\right)^{k_{2}-1} d u d v
$$

where $\gamma:=k_{1}+k_{2}, x=\left(x_{1}, x_{2}\right), y=\left(y_{1}, y_{2}\right)$,

$$
c_{k}=\frac{\Gamma\left(k_{1}+1 / 2\right) \Gamma\left(k_{2}+1 / 2\right)}{\pi \Gamma\left(k_{1}\right) \Gamma\left(k_{2}\right)},
$$

is the normalizing constant,

$$
\mathcal{I}_{\nu}(t)=\Gamma(\nu+1) \sum_{n=0}^{+\infty} \frac{(t / 2)^{2 n}}{n ! \Gamma(n+\nu+1)}, \quad t \in \mathbb{R} .
$$

is the normalized modified Bessel function of the first kind and of order $\nu$, and

$$
Z_{x, y}(u, v):=\left(x_{1}^{2}+x_{2}^{2}\right)\left(y_{1}^{2}+y_{2}^{2}\right)+u\left(x_{1}^{2}-x_{2}^{2}\right)\left(y_{1}^{2}-y_{2}^{2}\right)+4 v x_{1} x_{2} y_{1} y_{2} .
$$

Actually, (2.2) is a particular instance of a more general formula valid for integer values of $\gamma$, nevertheless it can extended to complex values with non negative real part using Carleson criteria as explained at the end of [10]. One can also see that (2.2) holds for non negative values of $\gamma$ by applying Lemma 8.5.2 in [18] to the Fourier-Gegenbauer series (3) in [11] (with the substitutions $\nu \rightarrow \gamma, p=2$ ).

\section{LAPlace-type integral Representation of the GBF of type $B_{2}$}

With the help of the notations introduced in the previous section, our main result may be stated as follows:

Theorem 1. If $\gamma>1 / 2$, then the GBF of type $B_{2}$ admits the following Laplace-type integral representation:

$$
D_{k}^{W}(x, y)=\int_{\mathbb{R}^{2}} e^{\langle x, z\rangle} H_{k}(y, z) d z
$$

where

$$
\begin{gathered}
H_{k}(y, z):=\frac{(2 \gamma-1) c_{k}}{\pi} \int_{E_{y, z}}\left(\left(y_{1}^{2}-y_{2}^{2}\right)^{2}\left(1-u^{2}\right)+4 y_{1}^{2} y_{2}^{2}\left(1-v^{2}\right)\right)^{-\gamma+1} \\
\left\{\left(y_{1}^{2}+y_{2}^{2}-\left(z_{1}^{2}+z_{2}^{2}\right)\right)^{2}-\left(z_{1}^{2}-z_{2}^{2}-u\left(y_{1}^{2}-y_{2}^{2}\right)\right)^{2}-4\left(z_{1} z_{2}-v y_{1} y_{2}\right)^{2}\right\}^{\gamma-3 / 2} \quad\left(1-u^{2}\right)^{k_{1}-1}\left(1-v^{2}\right)^{k_{2}-1} d u d v
\end{gathered}
$$

and

$$
E_{y, z}:=\left\{(u, v) \in[-1,1]^{2} ;\left(z_{1}^{2}-z_{2}^{2}-u\left(y_{1}^{2}-y_{2}^{2}\right)\right)^{2}+4\left(z_{1} z_{2}-v y_{1} y_{2}\right)^{2} \leq\left(y_{1}^{2}+y_{2}^{2}-\left(z_{1}^{2}+z_{2}^{2}\right)\right)^{2}\right\} .
$$


Proof. Without loss of generality, we may assume $y \neq(0,0)$ since $D_{k}^{W}(x,(0,0))=1$ for all $x \in \mathbb{R}^{2}$. Now, set!

$$
\begin{aligned}
a & :=\sqrt{\frac{y_{1}^{2}+y_{2}^{2}+u\left(y_{1}^{2}-y_{2}^{2}\right)}{2}} \\
b & :=\frac{\sqrt{\left(y_{1}^{2}-y_{2}^{2}\right)^{2}\left(1-u^{2}\right)+4 y_{1}^{2} y_{2}^{2}\left(1-v^{2}\right)}}{y_{1}^{2}+y_{2}^{2}+u\left(y_{1}^{2}-y_{2}^{2}\right)} \\
c & :=\frac{2 v y_{1} y_{2}}{y_{1}^{2}+y_{2}^{2}+u\left(y_{1}^{2}-y_{2}^{2}\right)} .
\end{aligned}
$$

Then, we can check that

$$
\sqrt{\frac{Z_{x, y}(u, v)}{2}}=a \sqrt{\left(x_{1}+c x_{2}\right)^{2}+b^{2} x_{2}^{2}}
$$

which shows that for fixed $y$, the argument of the modified Bessel function displayed in (2.2) may be written as the Euclidean norm of a planar vector. Furthermore, we shall need the following lemma:

Lemma 1. For any $\nu>0$ and any $z=\left(z_{1}, z_{2}\right) \in \mathbb{R}^{2}$, we have

$$
\mathcal{I}_{\nu}(\|z\|)=\frac{\nu}{\pi} \int_{\{\|y\| \leq 1\}} e^{\langle z, y\rangle}\left(1-\|y\|^{2}\right)^{\nu-1} d y
$$

where $\|z\|=\sqrt{z_{1}^{2}+z_{2}^{2}}$.

Proof. Using the invariance of the Lebesgue measure under rotations and a polar coordinates variable change, we write:

$$
\int_{\{\|y\| \leq 1\}} e^{\langle z, y\rangle}\left(1-\|y\|^{2}\right)^{\nu-1} d y=\int_{0}^{1} \int_{0}^{2 \pi} e^{\|z\| s \cos \theta} s\left(1-s^{2}\right)^{\nu-1} d \theta d s
$$

Integrating with respect to $\theta$ and appealing to the integral representation ([22])

we get:

$$
\mathcal{I}_{\nu}(t)=\frac{\Gamma(\nu+1)}{2 \sqrt{\pi} \Gamma(\nu+1 / 2)} \int_{0}^{2 \pi} e^{t \cos \theta} \sin ^{2 \nu} \theta d \theta
$$

$$
\int_{\{\|y\| \leq 1\}} e^{\langle z, y\rangle}\left(1-\|y\|^{2}\right)^{\nu-1} d y=2 \pi \int_{0}^{1} \mathcal{I}_{0}(\|z\| s) s\left(1-s^{2}\right)^{\nu-1} d s .
$$

Expanding the modified Bessel function $\mathcal{I}_{0}$ and integrating termwise finishes the proof.

Using lemma 1 followed by an affine change of variables, we readily derive

$\mathcal{I}_{\gamma-1 / 2}\left(\sqrt{\frac{Z_{x, y}(u, v)}{2}}\right)=\frac{\gamma-1 / 2}{\pi} \int_{\{\|z\| \leq 1\}} e^{a x_{1} z_{1}+a x_{2}\left(c z_{1}+b z_{2}\right)}\left(1-\|z\|^{2}\right)^{\gamma-3 / 2} d z$

$$
=\frac{\gamma-1 / 2}{\pi} \int_{\left\{b^{2} z_{1}^{2}+\left(z_{2}-c z_{1}\right)^{2} \leq a^{2} b^{2}\right\}} e^{\langle x, z\rangle} a^{-2 \gamma+1} b^{-2 \gamma+2}\left(a^{2} b^{2}-b^{2} z_{1}^{2}-\left(z_{2}-c z_{1}\right)^{2}\right)^{\gamma-3 / 2} d z .
$$

Besides,

$$
\begin{aligned}
& a^{-2 \gamma+1} b^{-2 \gamma+2}\left(a^{2} b^{2}-b^{2} z_{1}^{2}-\left(z_{2}-c z_{1}\right)^{2}\right)^{\gamma-3 / 2}=2\left(\left(y_{1}^{2}-y_{2}^{2}\right)^{2}\left(1-u^{2}\right)+4 y_{1}^{2} y_{2}^{2}\left(1-v^{2}\right)\right)^{-\gamma+1} \\
& \left\{\left(y_{1}^{2}+y_{2}^{2}-\left(z_{1}^{2}+z_{2}^{2}\right)\right)^{2}-\left(z_{1}^{2}-z_{2}^{2}-u\left(y_{1}^{2}-y_{2}^{2}\right)\right)^{2}-4\left(z_{1} z_{2}-v y_{1} y_{2}\right)^{2}\right\}^{\gamma-3 / 2}
\end{aligned}
$$

which leads to the desired result.

From Corollary 3.3 in $[$, the support of $z \mapsto H(y, z)$ lies in the convex hull co $(y)$ of the orbit of $y$ under the action of $W$. This geometrical fact is directly proved in the following proposition.

Proposition 1. For any $y \neq(0,0)$, the density $z \mapsto H(y, z)$ is supported in:

$$
c o(y)=\left\{z=\left(z_{1}, z_{2}\right) ;\left|z_{1}\right|+\left|z_{2}\right| \leq\left|y_{1}\right|+\left|y_{2}\right| ; \max \left(\left|z_{1}\right|,\left|z_{2}\right|\right) \leq \max \left(\left|y_{1}\right|,\left|y_{2}\right|\right)\right\} .
$$

\footnotetext{
${ }^{1}$ We ommit the dependence of $a, b, c$ on $(y, u, v)$ for sake of simplicity.
} 
Proof. From the definition of $H_{k}(y, z)$, it is sufficient to prove that for fixed $y \neq 0, E_{y, z} \neq \emptyset \Rightarrow z \in \operatorname{co}(y)$. To this end, let $y_{C}$ and $z_{C}$ be the representatives of $y$ and $z$ in $\bar{C}$ and recall from [19], Lemma 3.3, that

$$
z \in \operatorname{co}(y) \quad \Leftrightarrow \quad y_{C}-z_{C} \in \sum_{\alpha \in S} \mathbb{R}_{+} \alpha .
$$

This characterization of the convex hull of $W y$ together with the obvious fact that

$$
y_{C}=\left(\max \left(\left|y_{1}\right|,\left|y_{2}\right|\right), \min \left(\left|y_{1}\right|,\left|y_{2}\right|\right)\right), \quad z_{C}=\left(\max \left(\left|z_{1}\right|,\left|z_{2}\right|\right), \min \left(\left|z_{1}\right|,\left|z_{2}\right|\right)\right),
$$

lead to the above description of $\operatorname{co}(y)$. Now, Assume $E_{y, z} \neq \emptyset$ and recall the definition:

$$
E_{y, z}=\left\{(u, v) \in[-1,1]^{2} ; b^{2} z_{1}^{2}+\left(z_{2}-c z_{1}\right)^{2} \leq a^{2} b^{2}\right\} .
$$

Then, we readily see that

$$
\left|z_{1}\right| \leq a=\sqrt{\frac{y_{1}^{2}+y_{2}^{2}+u\left(y_{1}^{2}-y_{2}^{2}\right)}{2}} \leq \max \left(\left|y_{1}\right|,\left|y_{2}\right|\right), \quad .
$$

We can also rewrite $E_{y, z}$ as

$$
E_{y, z}=\left\{(u, v) \in[-1,1]^{2} ;\left(b^{2}+c^{2}\right)\left(z_{1}-\frac{c}{b^{2}+c^{2}} z_{2}\right)^{2}+\frac{b^{2}}{\left(b^{2}+c^{2}\right)} z_{2}^{2} \leq a^{2} b^{2}\right\}
$$

whence we deduce the inequality

$$
\left|z_{2}\right| \leq a \sqrt{b^{2}+c^{2}}=\sqrt{\frac{y_{1}^{2}+y_{2}^{2}-u\left(y_{1}^{2}-y_{2}^{2}\right)}{2}} \leq \max \left(\left|y_{1}\right|,\left|y_{2}\right|\right) .
$$

In particular, $z_{1}^{2}+z_{2}^{2} \leq y_{1}^{2}+y_{2}^{2}$. Next, write

$$
\begin{gathered}
\left(y_{1}^{2}+y_{2}^{2}-\left(z_{1}^{2}+z_{2}^{2}\right)\right)^{2}-\left(z_{1}^{2}-z_{2}^{2}-u\left(y_{1}^{2}-y_{2}^{2}\right)\right)^{2}-4\left(z_{1} z_{2}-v y_{1} y_{2}\right)^{2} \\
=\left(y_{1}^{2}+y_{2}^{2}+2 v y_{1} y_{2}-\left(z_{1}-z_{2}\right)^{2}\right)\left(y_{1}^{2}+y_{2}^{2}-2 v y_{1} y_{2}-\left(z_{1}+z_{2}\right)^{2}\right) \\
-\left(z_{1}^{2}-z_{2}^{2}-u\left(y_{1}^{2}-y_{2}^{2}\right)\right)^{2}
\end{gathered}
$$

to see that

$$
\left(y_{1}^{2}+y_{2}^{2}+2 v y_{1} y_{2}-\left(z_{1}-z_{2}\right)^{2}\right)\left(y_{1}^{2}+y_{2}^{2}-2 v y_{1} y_{2}-\left(z_{1}+z_{2}\right)^{2}\right) \geq 0 .
$$

But

$$
\left(y_{1}^{2}+y_{2}^{2}+2 v y_{1} y_{2}-\left(z_{1}-z_{2}\right)^{2}\right)+\left(y_{1}^{2}+y_{2}^{2}-2 v y_{1} y_{2}-\left(z_{1}+z_{2}\right)^{2}\right)=2\left(y_{1}^{2}+y_{2}^{2}-\left(z_{1}^{2}+z_{2}^{2}\right)\right) \geq 0
$$

therefore

$$
y_{1}^{2}+y_{2}^{2}+2 v y_{1} y_{2}-\left(z_{1}-z_{2}\right)^{2} \geq 0 ; \quad y_{1}^{2}+y_{2}^{2}-2 v y_{1} y_{2}-\left(z_{1}+z_{2}\right)^{2} \geq 0 .
$$

Since $|v| \leq 1$, then

$$
\left|z_{1}-z_{2}\right| \leq\left|y_{1}\right|+\left|y_{2}\right| ; \quad\left|z_{1}+z_{2}\right| \leq\left|y_{1}\right|+\left|y_{2}\right|
$$

whence it follows that $\left|z_{1}\right|+\left|z_{2}\right| \leq\left|y_{1}\right|+\left|y_{2}\right|$ and the proposition is proved.

Remark 1. When $k_{0}=k_{1}=1$ and $y_{1}>y_{2}>0$ (or equivalently $y \in C$ ), the measure $H_{1}(y, z) d z$ reduces, up to a multiplicative factor, to the Duistermaat-Heckman measure associated with the group $W=\left(\mathbb{Z}_{2}\right)^{2} \rtimes S_{2}$ ([3]):

$$
\begin{aligned}
H_{1}(y, z) & =\frac{3}{4 \pi} \int_{E_{y, z}} \frac{\sqrt{\left(y_{1}^{2}+y_{2}^{2}-\left(z_{1}^{2}+z_{2}^{2}\right)\right)^{2}-\left(z_{1}^{2}-z_{2}^{2}-u\left(y_{1}^{2}-y_{2}^{2}\right)\right)^{2}-4\left(z_{1} z_{2}-v y_{1} y_{2}\right)^{2}}}{\left(y_{1}^{2}-y_{2}^{2}\right)^{2}\left(1-u^{2}\right)+4 y_{1}^{2} y_{2}^{2}\left(1-v^{2}\right)} d u d v \\
& =\frac{3}{4 \pi\left(y_{1}^{2}-y_{2}^{2}\right) y_{1} y_{2}} \int_{F_{y, z}} \frac{\sqrt{\left(y_{1}^{2}+y_{2}^{2}-\left(z_{1}^{2}+z_{2}^{2}\right)\right)^{2}-\left(z_{1}^{2}-z_{2}^{2}-u\right)^{2}-\left(2 z_{1} z_{2}-v\right)^{2}}}{\left(y_{1}^{2}+y_{2}^{2}\right)^{2}-u^{2}-v^{2}} d u d v \\
& =\frac{1}{2\left(y_{1}^{2}-y_{2}^{2}\right) y_{1} y_{2}} m_{D H}(y, d z)
\end{aligned}
$$

where

$$
F_{y, z}:=\left\{|u|<y_{1}^{2}-y_{2}^{2},|v|<2 y_{1} y_{2} ;\left(z_{1}^{2}-z_{2}^{2}-u\right)^{2}+\left(2 z_{1} z_{2}-v\right)^{2} \leq\left(y_{1}^{2}+y_{2}^{2}-\left(z_{1}^{2}+z_{2}^{2}\right)\right)^{2}\right\} .
$$


Moreover, the former is the conditional distribution of the value of a planar Brownian motion at a fixed time $T>0$ given that the value of the generalized Pitman transform of this process at time $T$ is $y$, while the latter is the push forward of the Lebesgue measure of some (string) polytope $\mathcal{P}$ in $\mathbb{R}^{4}$ under an affine map. In suitable coordinates, $\mathcal{P}$ transforms into the Gelfand-Tsetlin pattern of $s p(4, \mathbb{C}$ ) at $y$ defined by (see e.g. [4], 20]):

$$
G T_{s p(4, \mathbb{C})}:=\left\{\left(w_{1}, w_{2}, t\right), y_{1} \geq w_{1} \geq y_{2} \geq w_{2} \geq 0, w_{1} \geq t \geq w_{2}\right\} .
$$

Note that for the A-type root system or corresponding to the Lie algebra sl( $N, \mathbb{C})$, the Gelfand-Tsetlin pattern shows up directly in the inductive construction of the GBF since a similar one already exists for Jack polynomials ([1]).

\section{Laplace-type integral Representation of the Dunkl Kernel of type $B_{2}$}

Using Theorem 1, we shall derive a Laplace-type integral representation for the Dunkl kernel $D_{k}$ associated with the root system of type $B_{2}$. To this end, we appeal to the so-called Shift principle which we briefly outline (see [14, Prop. 1.4). Let $\mathcal{C}$ be an orbit of the action of $W$ on $R$ and let $k$ be a nonnegative multiplicity function. Then

$$
k^{\prime}:=k+\mathbf{1}_{\mathcal{C}}
$$

is the shifted multiplicity function on $\mathcal{C}$ and the following holds:

$$
\sum_{w \in W} \chi_{\mathcal{C}}(w) D_{k}(x, w y)=d_{\mathcal{C}}(k) p_{\mathcal{C}}(x) p_{\mathcal{C}}(y) D_{k^{\prime}}^{W}(x, y), \quad x, y \in C
$$

where

$$
p_{\mathcal{C}}(x):=\prod_{\alpha \in \mathcal{C} \cap R_{+}}\langle\alpha, x\rangle,
$$

is the alternating polynomial associated with $\mathcal{C}, \chi_{\mathcal{C}}$ is its linear character defined by:

$$
p_{\mathcal{C}}(w x)=\chi_{\mathcal{C}}(w) p_{\mathcal{C}}(x), \quad w \in W,
$$

and $d_{k}$ is the normalizing constant given by

$$
d_{\mathcal{C}}(k):=\frac{|W|}{p_{\mathcal{C}}\left(T_{1}, \ldots, T_{N}\right) p_{\mathcal{C}}} .
$$

This principle was already used in 2] to derive the Laplace-type integral representation of the Dunkl kernel associated with the $A_{2}$-type root system and in 8 in a similar fashion to get an integral representation of $D_{k}$ associated with the $B_{2}$-type root system. Nonetheless, the representation we prove below improves the one derived in [8] since it is of Laplace-type. In order to state it, we shall apply the shift principle to the orbits

$$
\mathcal{C}_{1}=\left\{ \pm\left(e_{1}-e_{2}\right), \pm\left(e_{1}+e_{2}\right)\right\}, \quad \mathcal{C}_{2}=\left\{ \pm e_{1}, \pm e_{2}\right\}
$$

to which assigned the multiplicity values $k_{1}, k_{2}$ respectively, and to the whole root system $R$ as well. Consequently, the corresponding alternating polynomials are given by

$$
S_{1}(x):=p_{\mathcal{C}_{1}}(x)=x_{1}^{2}-x_{2}^{2}, \quad S_{2}(x):=p_{\mathcal{C}_{2}}(x)=x_{1} x_{2}, \quad S_{3}(x):=p_{R}(x)=x_{1} x_{2}\left(x_{1}^{2}-x_{2}^{2}\right),
$$

whence we easily derive the following expression:2:

$$
\begin{aligned}
& d_{1}(k):=d_{\mathcal{C}_{1}}(k)=\frac{2}{\left(2 k_{1}+1\right)(2 \gamma+1)}, \\
& d_{2}(k):=d_{\mathcal{C}_{2}}(k)=\frac{8}{\left(2 k_{2}+1\right)(2 \gamma+1)}, \\
& d_{3}(k):=d_{R}(k)=\frac{1}{2\left(2 k_{1}+1\right)\left(2 k_{2}+1\right)(2 \gamma+1)(2 \gamma+3)} .
\end{aligned}
$$

With these findings, we prove the following:

\footnotetext{
${ }^{2}$ See [16, p. 249, for the expression of $d_{3}(k)$.
} 
Proposition 2. The Dunkl kernel and the GBF of type $B_{2}$ are interrelated by

$$
\begin{aligned}
2 y_{1} D_{k}(x, y) & =\left(T_{1}+y_{1}\right)\left\{\frac { 1 } { 4 } \left(d_{1}(k) S_{1}(x) S_{1}(y) D_{\left(k_{1}+1, k_{2}\right)}^{W}(x, y)\right.\right. \\
& \left.\left.+d_{2}(k) S_{2}(x) S_{2}(y) D_{\left(k_{1}, k_{2}+1\right)}^{W}(x, y)+d_{3}(k) S_{3}(x) S_{3}(y) D_{\left(k_{1}+1, k_{2}+1\right)}^{W}(x, y)\right)+2 D_{k}^{W}(x, y)\right\},
\end{aligned}
$$

where we write $D_{k}^{W}=D_{\left(k_{1}, k_{2}\right)}^{W}$ in order to emphasize the shift action of $k=\left(k_{1}, k_{2}\right)$ and $T_{1}$ is the Dunkl derivative in the direction $e_{1}$ acting on $x$.

Proof. Recall that the Weyl group $W=\left(\mathbb{Z}_{2}\right)^{2} \rtimes S_{2}$ consists of the eight elements below:

$$
\begin{gathered}
\sigma_{1}=\left(\begin{array}{cc}
-1 & 0 \\
0 & 1
\end{array}\right), \sigma_{2}=\left(\begin{array}{cc}
1 & 0 \\
0 & -1
\end{array}\right), \sigma_{3}=\left(\begin{array}{ll}
0 & 1 \\
1 & 0
\end{array}\right), \sigma_{4}=\left(\begin{array}{cc}
0 & -1 \\
-1 & 0
\end{array}\right), \\
r=\left(\begin{array}{cc}
0 & 1 \\
-1 & 0
\end{array}\right), r^{2}=\left(\begin{array}{cc}
-1 & 0 \\
0 & -1
\end{array}\right), r^{3}=\left(\begin{array}{cc}
0 & -1 \\
1 & 0
\end{array}\right), i d=\left(\begin{array}{cc}
1 & 0 \\
0 & 1
\end{array}\right) .
\end{gathered}
$$

Applying the Shift principle to $\mathcal{C}_{1}, \mathcal{C}_{2}, R$ respectively, we derive the following identities:

$$
\begin{aligned}
& D_{k}\left(x, \sigma_{1} y\right)+D_{k}\left(x, \sigma_{2} y\right)-D_{k}\left(x, \sigma_{3} y\right)-D_{k}\left(x, \sigma_{4} y\right)-D_{k}(x, r y)+D_{k}\left(x, r^{2} y\right) \\
& -D_{k}\left(x, r^{3} y\right)+D_{k}(x, y)=d_{1}(k) S_{1}(x) S_{1}(y) D_{\left(k_{1}+1, k_{2}\right)}^{W}(x, y), \\
& -D_{k}\left(x, \sigma_{1} y\right)-D_{k}\left(x, \sigma_{2} y\right)+D_{k}\left(x, \sigma_{3} y\right)+D_{k}\left(x, \sigma_{4} y\right)-D_{k}(x, r y)+D_{k}\left(x, r^{2} y\right) \\
& -D_{k}\left(x, r^{3} y\right)+D_{k}(x, y)=d_{2}(k) S_{2}(x) S_{2}(y) D_{\left(k_{1}, k_{2}+1\right)}^{W}(x, y),
\end{aligned}
$$

and

$$
\begin{aligned}
& -D_{k}\left(x, \sigma_{1} y\right)-D_{k}\left(x, \sigma_{2} y\right)-D_{k}\left(x, \sigma_{3} y\right)-D_{k}\left(x, \sigma_{4} y\right)+D_{k}(x, r y)+D_{k}\left(x, r^{2} y\right) \\
& +D_{k}\left(x, r^{3} y\right)+D_{k}(x, y)=d_{3}(k) S_{3}(x) S_{3}(y) D_{\left(k_{1}+1, k+1\right)}^{W}(x, y),
\end{aligned}
$$

Together with (2.1), they lead to

$$
\begin{aligned}
& D_{k}(x, y)+D_{k}(x,-y)=\frac{1}{4}\left(d_{1}(k) S_{1}(x) S_{1}(y) D_{\left(k_{1}+1, k_{2}\right)}^{W}(x, y)\right. \\
& \left.+d_{2}(k) S_{2}(x) S_{2}(y) D_{\left(k_{1}, k_{2}+1\right)}^{W}(x, y)+d_{3}(k) S_{3}(x) S_{3}(y) D_{\left(k_{1}+1, k_{2}+1\right)}^{W}(x, y)\right)+2 D_{k}^{W}(x, y)
\end{aligned}
$$

Since $T_{1} D_{k}(\cdot, y)(x)=y_{1} D_{k}(x, y)$, then the proposition follows.

With regard to proposition 2 we need to work out the right hand side of (4.1). The issue of our computations is recorded in the corollary below:

Corollary 1. If $\gamma=k_{0}+k_{1}>1 / 2$, then the Dunkl kernel of type $B_{2}$ admits the following Laplace-type integral representation:

$$
2 y_{1} D_{k}(x, y)=\int_{c o(y)} e^{\langle x, z\rangle} L_{k}(y, z) d z
$$

where

$$
\begin{aligned}
& L_{k}(y, z):=\int_{E_{y, z}}\left\{2\left(z_{1}+y_{1}\right)(4 u+4 v+u v+4)+2 \frac{S_{2}(y)}{(a b)^{2}}\left(z_{2}-c z_{1}\right)(u+4)\left(1-v^{2}\right)\right. \\
+ & \left.\frac{S_{1}(y)}{(a b)^{2}}\left(\left(b^{2}+c^{2}\right) z_{1}-c z_{2}\right)(v+4)\left(1-u^{2}\right)\right\} \frac{\left(a^{2} b^{2}-b^{2} z_{1}^{2}-\left(z_{2}-c z_{1}\right)^{2}\right)^{\gamma-3 / 2}}{a^{2 \gamma-1} b^{2 \gamma-2}}\left(1-u^{2}\right)^{k_{1}-1}\left(1-v^{2}\right)^{k_{2}-1} d z d u d v .
\end{aligned}
$$

Proof. Write (2.3) as

$$
Z_{x, y}(u, v)=\left(x_{1}^{2}+x_{2}^{2}\right)\left(y_{1}^{2}+y_{2}^{2}\right)+u S_{1}(x) S_{1}(y)+4 v S_{2}(x) S_{2}(y) .
$$

Then, the differentiation rule

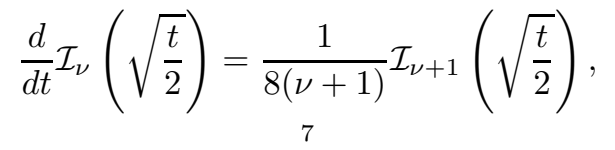


and (2.2) yield:

$$
\begin{aligned}
d_{1}(k) S_{1}(x) S_{1}(y) D_{\left(k_{1}+1, k_{2}\right)}^{W}(x, y) & =\frac{4 c_{k}}{k_{1}} \int_{-1}^{1} \int_{-1}^{1} \frac{\partial}{\partial u}\left\{\mathcal{I}_{\gamma-1 / 2}\left(\sqrt{\frac{Z_{x, y}(u, v)}{2}}\right)\right\}\left(1-u^{2}\right)^{k_{1}}\left(1-v^{2}\right)^{k_{2}-1} d u d v \\
& =8 c_{k} \int_{-1}^{1} \int_{-1}^{1} \mathcal{I}_{\gamma-1 / 2}\left(\sqrt{\frac{Z_{x, y}(u, v)}{2}}\right) u\left(1-u^{2}\right)^{k_{1}-1}\left(1-v^{2}\right)^{k_{2}-1} d u d v .
\end{aligned}
$$

Similarly,

$$
\begin{aligned}
d_{2}(k) S_{2}(x) S_{2}(y) D_{\left(k_{1}, k_{2}+1\right)}^{W}(x, y) & =\frac{4 c_{k}}{k_{2}} \int_{-1}^{1} \int_{-1}^{1} \frac{\partial}{\partial v}\left\{\mathcal{I}_{\gamma-1 / 2}\left(\sqrt{\frac{Z_{x, y}(u, v)}{2}}\right)\right\}\left(1-u^{2}\right)^{k_{1}-1}\left(1-v^{2}\right)^{k_{2}} d u d v \\
& =8 c_{k} \int_{-1}^{1} \int_{-1}^{1} \mathcal{I}_{\gamma-1 / 2}\left(\sqrt{\frac{Z_{x, y}(u, v)}{2}}\right) v\left(1-v^{2}\right)^{k_{2}-1}\left(1-u^{2}\right)^{k_{1}-1} d u d v
\end{aligned}
$$

and

$$
\begin{aligned}
d_{3}(k) S_{3}(x) S_{3}(y) D_{\left(k_{1}+1, k_{2}+1\right)}^{W}(x, y) & =\frac{c_{k}}{2 k_{1} k_{2}} \int_{-1}^{1} \int_{-1}^{1} \frac{\partial^{2}}{\partial u \partial v}\left\{\mathcal{I}_{\gamma-1 / 2}\left(\sqrt{\frac{Z_{x, y}(u, v)}{2}}\right)\right\}\left(1-u^{2}\right)^{k_{1}}\left(1-v^{2}\right)^{k_{2}} d u d v \\
& =2 c_{k} \int_{-1}^{1} \int_{-1}^{1} \mathcal{I}_{\gamma-1 / 2}\left(\sqrt{\frac{Z_{x, y}(u, v)}{2}}\right) u v\left(1-u^{2}\right)^{k_{1}-1}\left(1-v^{2}\right)^{k_{2}-1} d u d v .
\end{aligned}
$$

Altogether yield

$$
\begin{aligned}
& \frac{1}{4}\left(d_{1}(k) S_{1}(x) S_{1}(y) D_{\left(k_{1}+1, k_{2}\right)}^{W}(x, y)+d_{2}(k) S_{2}(x) S_{2}(y) D_{\left(k_{1}, k_{2}+1\right)}^{W}(x, y)\right. \\
&\left.+d_{3}(k) S_{3}(x) S_{3}(y) D_{\left(k_{1}+1, k_{2}+1\right)}^{W}(x, y)\right)+2 D_{k}^{W}(x, y) \\
&= \frac{c_{k}}{2} \int_{-1}^{1} \int_{-1}^{1} \mathcal{I}_{\gamma-1 / 2}\left(\sqrt{\frac{Z_{x, y}(u, v)}{2}}\right)(4 u+4 v+u v+4)\left(1-u^{2}\right)^{k_{1}-1}\left(1-v^{2}\right)^{k_{2}-1} d u d v \\
&= \frac{(2 \gamma-1) c_{k}}{4 \pi} \int_{-1}^{1} \int_{-1}^{1} \int_{\{\|z\| \leq 1\}} e^{a x_{1} z_{1}+a x_{2}\left(c z_{1}+b z_{2}\right)}\left(1-\|z\|^{2}\right)^{\gamma-3 / 2}(4 u+4 v+u v+4) \\
&\left(1-u^{2}\right)^{k_{1}-1}\left(1-v^{2}\right)^{k_{2}-1} d z d u d v .
\end{aligned}
$$

Next, recall the expression of the Dunkl derivative $T_{1}$ in the direction $e_{1}$ acting on $x$ :

$$
\begin{aligned}
T_{1} f(x) & =\partial_{1} f(x)+\sum_{\alpha \in R_{+}} k(\alpha)\left\langle\alpha, e_{1}\right\rangle \frac{f(x)-f\left(\sigma_{\alpha} x\right)}{\langle\alpha, x\rangle} \\
& =\partial_{1} f(x)+k_{2} \frac{f(x)-f\left(\sigma_{1} x\right)}{x_{1}}+k_{1}\left\{\frac{f(x)-f\left(\sigma_{3} x\right)}{x_{1}-x_{2}}+\frac{f(x)-f\left(\sigma_{4} x\right)}{x_{1}+x_{2}}\right\} .
\end{aligned}
$$

Then, the invariance of $D_{k}^{W}$ under the action of $W$ entails:

$$
\begin{aligned}
& \text { (4.3) } T_{1}\left\{\frac { 1 } { 4 } \left(d_{1}(k) S_{1}(x) S_{1}(y) D_{\left(k_{1}+1, k_{2}\right)}^{W}(x, y)+d_{2}(k) S_{2}(x) S_{2}(y) D_{\left(k_{1}, k_{2}+1\right)}^{W}(x, y)\right.\right. \\
& \left.\left.+d_{3}(k) S_{3}(x) S_{3}(y) D_{\left(k_{1}+1, k_{2}+1\right)}^{W}(x, y)\right)+2 D_{k}^{W}(x, y)\right\}=\frac{(2 \gamma-1) c_{k}}{4 \pi} \int_{-1}^{1} \int_{-1}^{1} \int_{\{\|z\| \leq 1\}} a z_{1} e^{a x_{1} z_{1}+a x_{2}\left(c z_{1}+b z_{2}\right)} \\
& \left(1-\|z\|^{2}\right)^{\gamma-3 / 2}(4 u+4 v+u v+4)\left(1-u^{2}\right)^{k_{1}-1}\left(1-v^{2}\right)^{k_{2}-1} d z d u d v+I_{2}(x, y)
\end{aligned}
$$

where we set

$$
\begin{aligned}
I_{2}(x, y):=d_{1}(k) & S_{1}(y)\left(k_{1} x_{1}\right) D_{\left(k_{1}+1, k_{2}\right)}^{W}(x, y)+d_{2}(k) S_{2}(y) \frac{k_{2} x_{2}}{2} D_{\left(k_{1}, k_{2}+1\right)}^{W}(x, y) \\
& +d_{3}(k) S_{3}(y) S_{1}(x) \frac{k_{2} x_{2}}{2} D_{\left(k_{1}+1, k_{2}+1\right)}^{W}(x, y)+d_{3}(k) S_{3}(y) S_{2}(x)\left(k_{1} x_{1}\right) D_{\left(k_{1}+1, k_{2}+1\right)}^{W}(x, y) .
\end{aligned}
$$


Now, it remains to put $I_{2}(x, y)$ in form of (3.2). To proceed, we first use Lemma 1 and integration by parts to derive

$$
\begin{aligned}
x_{2} \mathcal{I}_{\gamma+1 / 2}\left(\sqrt{\frac{Z_{x, y}(u, v)}{2}}\right) & =\frac{2 \gamma+1}{2 \pi} \int_{\{\|z\| \leq 1\}} x_{2} e^{a x_{1} z_{1}+a x_{2}\left(c z_{1}+b z_{2}\right)}\left(1-\|z\|^{2}\right)^{\gamma-1 / 2} d z \\
& =\frac{(2 \gamma+1)(2 \gamma-1)}{2 \pi} \int_{\{\|z\| \leq 1\}} \frac{z_{2}}{a b} e^{a x_{1} z_{1}+a x_{2}\left(c z_{1}+b z_{2}\right)}\left(1-\|z\|^{2}\right)^{\gamma-3 / 2} d z
\end{aligned}
$$

and

$$
x_{1} \mathcal{I}_{\gamma+1 / 2}\left(\sqrt{\frac{Z_{x, y}(u, v)}{2}}\right)=\frac{(2 \gamma+1)(2 \gamma-1)}{2 \pi} \int_{\{\|z\| \leq 1\}}\left(\frac{z_{1}}{a}-\frac{c z_{2}}{a b}\right) e^{a x_{1} z_{1}+a x_{2}\left(c z_{1}+b z_{2}\right)}\left(1-\|z\|^{2}\right)^{\gamma-1 / 2} d z .
$$

It follows that

$$
\begin{aligned}
\frac{k_{2} x_{2}}{2} d_{2}(k) S_{2}(y) D_{\left(k_{1}, k_{2}+1\right)}^{W}(x, y)=\frac{(2 \gamma-1) c_{k}}{\pi} S_{2}(y) & \int_{-1}^{1} \int_{-1}^{1} \int_{\{\|z\| \leq 1\}} \frac{z_{2}}{a b} e^{a x_{1} z_{1}+a x_{2}\left(c z_{1}+b z_{2}\right)} \\
& \left(1-\|z\|^{2}\right)^{\gamma-3 / 2}\left(1-u^{2}\right)^{k_{1}-1}\left(1-v^{2}\right)^{k_{2}} d z d u d v
\end{aligned}
$$

and

$$
\begin{array}{r}
d_{1}(k) S_{1}(y)\left(k_{1} x_{1}\right) D_{\left(k_{1}+1, k_{2}\right)}^{W}(x, y)=\frac{(2 \gamma-1) c_{k}}{2 \pi} S_{1}(y) \int_{-1}^{1} \int_{-1}^{1} \int_{\{\|z\| \leq 1\}}\left(\frac{z_{1}}{a}-\frac{c z_{2}}{a b}\right) e^{a x_{1} z_{1}+a x_{2}\left(c z_{1}+b z_{2}\right)} \\
\left(1-\|z\|^{2}\right)^{\gamma-3 / 2}\left(1-u^{2}\right)^{k_{1}}\left(1-v^{2}\right)^{k_{2}-1} d z d u d v
\end{array}
$$

Besides, the differentiation rule (4.2), (4.4) and (4.5) yield

$$
\begin{gathered}
\int_{-1}^{1} \int_{-1}^{1} x_{1} \frac{\partial}{\partial v}\left\{\mathcal{I}_{\gamma+1 / 2}\left(\sqrt{\frac{Z_{x, y}(u, v)}{2}}\right)\right\}\left(1-u^{2}\right)^{k_{1}}\left(1-v^{2}\right)^{k_{2}} d u d v \\
=\frac{d_{3}(k)\left(2 k_{1}+1\right)\left(2 k_{2}+1\right)(2 \gamma+3) c_{k} S_{1}(y)}{2} \int_{-1}^{1} \int_{-1}^{1} x_{1} \mathcal{I}_{\gamma+1 / 2}\left(\sqrt{\frac{Z_{x, y}(u, v)}{2}}\right) v\left(1-v^{2}\right)^{k_{2}-1}\left(1-u^{2}\right)^{k_{1}} d u d v \\
=\frac{(2 \gamma-1) c_{k}}{8 \pi} S_{1}(y) \int_{-1}^{1} \int_{-1}^{1} \int_{\{\|z\| \leq 1\}}\left(\frac{z_{1}}{a}-\frac{c z_{2}}{a b}\right) e^{a x_{1} z_{1}+a x_{2}\left(c z_{1}+b z_{2}\right)} \\
\left(1-\|z\|^{2}\right)^{\gamma-1 / 2} v\left(1-v^{2}\right)^{k_{2}-1}\left(1-u^{2}\right)^{k_{1}} d z d u d v
\end{gathered}
$$

and

$$
\begin{aligned}
& \text { (4.9) } d_{3}(k) \frac{k_{2} x_{2}}{2} S_{1}(x) S_{3}(y) D_{\left(k_{1}+1, k_{2}+1\right)}^{W}(x, y)=\frac{d_{3}(k)\left(2 k_{1}+1\right)\left(2 k_{2}+1\right)(2 \gamma+3) c_{k}}{2 k_{1}} S_{2}(y) \\
& \qquad \int_{-1}^{1} \int_{-1}^{1} x_{2} \frac{\partial}{\partial u}\left\{\mathcal{I}_{\gamma+1 / 2}\left(\sqrt{\frac{Z_{x, y}(u, v)}{2}}\right)\right\}\left(1-u^{2}\right)^{k_{1}}\left(1-v^{2}\right)^{k_{2}} d u d v \\
& =d_{3}(k)\left(2 k_{1}+1\right)\left(2 k_{2}+1\right)(2 \gamma+3) S_{2}(y) \int_{-1}^{1} \int_{-1}^{1} x_{2} \mathcal{I}_{\gamma+1 / 2}\left(\sqrt{\frac{Z_{x, y}(u, v)}{2}}\right) u\left(1-u^{2}\right)^{k_{1}-1}\left(1-v^{2}\right)^{k_{2}} d u d v \\
& =\frac{(2 \gamma-1) c_{k}}{4 \pi} S_{2}(y) \int_{-1}^{1} \int_{-1}^{1} \int_{\{\|z\| \leq 1\}} \frac{z_{2}}{a b} e^{a x_{1} z_{1}+a x_{2}\left(c z_{1}+b z_{2}\right)}\left(1-\|z\|^{2}\right)^{\gamma-3 / 2} u\left(1-u^{2}\right)^{k_{1}-1}\left(1-v^{2}\right)^{k_{2}} d z d u d v .
\end{aligned}
$$


Gathering (4.6), (4.7), (4.8), (4.9), we get

$$
\begin{array}{r}
I_{2}(x, y)=\frac{(2 \gamma-1) c_{k}}{8 \pi} \int_{-1}^{1} \int_{-1}^{1} \int_{\{\|z\| \leq 1\}}\left\{2 S_{2}(y) \frac{z_{2}}{a b}(u+4)\left(1-v^{2}\right)+S_{1}(y)\left(\frac{z_{1}}{a}-\frac{c z_{2}}{a b}\right)(v+4)\left(1-u^{2}\right)\right\} \\
\quad e^{a x_{1} z_{1}+a x_{2}\left(c z_{1}+b z_{2}\right)}\left(1-\|z\|^{2}\right)^{\gamma-3 / 2}\left(1-u^{2}\right)^{k_{1}-1}\left(1-v^{2}\right)^{k_{2}-1} d z d u d v .
\end{array}
$$

Keeping in mind (4.3), we get:

$$
T_{1}\left\{\frac { 1 } { 4 } \left(d_{1}(k) S_{1}(x) S_{1}(y) D_{\left(k_{1}+1, k_{2}\right)}^{W}(x, y)+d_{2}(k) S_{2}(x) S_{2}(y) D_{\left(k_{1}, k_{2}+1\right)}^{W}(x, y)+\right.\right.
$$

$$
\left.\left.d_{3}(k) S_{3}(x) S_{3}(y) D_{\left(k_{1}+1, k_{2}+1\right)}^{W}(x, y)\right)+2 D_{k}^{W}(x, y)\right\}
$$

$$
\begin{aligned}
& =\frac{(2 \gamma-1) c_{k}}{8 \pi} \int_{-1}^{1} \int_{-1}^{1} \int_{\{\|z\| \leq 1\}}\left\{2 a z_{1}(4 u+4 v+u v+4)+2 S_{2}(y) \frac{z_{2}}{a b}(u+4)\left(1-v^{2}\right)+S_{1}(y)\left(\frac{z_{1}}{a}-\frac{c z_{2}}{a b}\right)(v+4)\left(1-u^{2}\right)\right\} \\
& e^{a x_{1} z_{1}+a x_{2}\left(c z_{1}+b z_{2}\right)}\left(1-\|z\|^{2}\right)^{\gamma-3 / 2}\left(1-u^{2}\right)^{k_{1}-1}\left(1-v^{2}\right)^{k_{2}-1} d z d u d v .
\end{aligned}
$$

Consequently, the previous proposition implies

$$
\begin{aligned}
& 2 y_{1} D_{k}(x, y)=\frac{(2 \gamma-1) c_{k}}{8 \pi} \int_{-1}^{1} \int_{-1}^{1} \int_{\{\|z\| \leq 1\}}\left\{2\left(a z_{1}+y_{1}\right)(4 u+4 v+u v+4)+2 S_{2}(y) \frac{z_{2}}{a b}(u+4)\left(1-v^{2}\right)\right. \\
& \left.+S_{1}(y)\left(\frac{z_{1}}{a}-\frac{c z_{2}}{a b}\right)(v+4)\left(1-u^{2}\right)\right\} e^{a x_{1} z_{1}+a x_{2}\left(c z_{1}+b z_{2}\right)}\left(1-\|z\|^{2}\right)^{\gamma-3 / 2}\left(1-u^{2}\right)^{k_{1}-1}\left(1-v^{2}\right)^{k_{2}-1} d z d u d v .
\end{aligned}
$$

Performing the variables change

$$
a z_{1} \rightarrow z_{1}, \quad a\left(c z_{1}+b z_{2}\right) \rightarrow z_{2},
$$

we finally get the formula displayed in the corollary.

\section{REFERENCES}

[1] B. Amri. Note on Bessel functions of type $A_{N-1}$. Integ. Transf. Spec. Funct. 25, No. 4, (2014). 448-461.

[2] B. Amri. On the Integral Representations for Dunkl Kernels of Type A2. J. Lie Theory. 26. (2016), No. 4, 1163-1175.

[3] P. Biane, P. Bougerol, N. O'Connell. Continuous crystals and Duistermaat-Heckman measure for Coxeter groups, Advances in Mathematics. 221 (2009), 1522-1583.

[4] A. D. Berenstein, A. V. Zelevinsky. Tensor product multiplicities and convex polytopes in partition space. J. Geom. Phys. 5 (1988), no. 3, 453-472.

[5] O. Chybiryakov, N. Demni, L. Gallardo, M. Rösler, M. Voit, M. Yor. Harmonic and Stochastic Analysis of Dunkl Processes. Ed. P. Graczyk, M. Rösler, M. Yor, Collection Travaux en Cours, Hermann.

[6] M. F. E. de Jeu, The Dunkl transform. Invent. Math. 113 (1993), 147-162.

[7] M. Defosseux. Orbit measures, random matrix theory and interlaced determinantal processes. Ann. Inst. Henri Poincaré Probab. Stat. 46 (2010), no. 1, 209-249.

[8] L. Deléaval, N. Demni, H. Youssfi. Dunkl kernel associated with dihedral systems. Dunkl kernel associated with dihedral groups. J. Math. Anal. Appl. 432, (2015), no. 2, 928-944.

[9] N. Demni. Radial Dunkl processes associated with dihedral systems. Séminaire de probabilités XLII, 153-169, Lecture Notes in Math., 1979, Springer, Berlin, 2009.

[10] N. Demni. Product formula for Jacobi polynomials, spherical harmonics and generalized Bessel function of dihedral type. Integral Transforms Spec. Funct. 21, (2010), no. 1-2, 105-123.

[11] N. Demni. Generalized Bessel function associated with dihedral groups. J. Lie Theory. 22 (2012), no. 1, 81-91.

[12] N. Demni, D. Lépingle. Brownian motion, reflection groups and Tanaka formula. Rend. Semin. Mat. Univ. Padova. 127 (2012), 41-55.

[13] C. F. Dunkl. Differential-difference operators associated to reflection groups. Trans. Amer. Math. Soc. 311 (1989), no. 1, $167-183$.

[14] C. F. Dunkl. Intertwining operators associated to the group S3. Trans. Amer. Math. Soc. 347 (1995), no. 9, $3347-3374$.

[15] C. F. Dunkl. An intertwining operator for the group B2. Glasg. Math. J. 49 (2007), no. 2, 291-319.

[16] C. F. Dunkl, M. F. E. De Jeu, E. M. Opdam. Singular polynomials for finite reflection groups. Trans. Amer. Math. Soc. 346 (1994), no. 1, 237-256.

[17] C.F. Dunkl, Y. Xu. Orthogonal Polynomials of Several Variables, Encyclopedia Math. Appl., Cambridge University Press, 2001. 
[18] T. Kobayashi, G. Mano. The inversion formula and holomorphic extension of the minimal representation of the conformal group. Harmonic analysis, group representations, automorphic forms and invariant theory, 151-208, Lect. Notes Ser. Inst. Math. Sci. Natl. Univ. Singap., 12, World Sci. Publ., Hackensack, NJ, 2007.

[19] B. Kostant. On convexity, the Weyl group and the Iwasawa decomposition. Ann. Sci. cole Norm. Sup. 4, (6) (1973), 413-455, (1974).

[20] A. Okounkov. Multiplicities and Newton polytopes. Kirillov's seminar on representation theory, 231-244, Amer. Math. Soc. Transl. Ser. 2, 181, Amer. Math. Soc., Providence, RI, 1998.

[21] M. Rösler. Positivity of Dunkl's intertwining operator. Duke Math. J. 98 (1999), 445-463.

[22] G. N. Watson. A treatise on the theory of Bessel functions. Second Edition. Cambridge Mathematical Library edition, (1962).

[23] Y. Xu. A product formula for Jacobi polynomials. in Special Functions, Proceedings of International Workshop, Hong Kong, June 21-25, (1999), World Sci. Publ., 2000, p. 423-430.

Université Tunis El Manar, Faculté des sciences de Tunis, Laboratoire d’Analyse Mathématique et ApplicaTiOns, LR11ES11, 2092 El Manar I, Tunisie

E-mail address: bechir.amri@gmail.com

IRMAR, Université de Rennes 1, Campus de Beaulieu, 35042 Rennes Cedex, France

E-mail address: nizar.demni@univ-rennes1.fr 